\title{
Grid-Shadow Effect in Grid-Enhanced Plasma Source Ion Implantation
}

\author{
J.L. Wang, G.L. Zhang, Y.F. Liu, X.M. Li, W.R. Feng, G.L. Chen, C.Z. Liu, S.Z. Yang \\ Institute of Physics, Chinese Academy of Sciences, Beijing 100080, China \\ Phone: +86-10-8264-9458; Fax: +86-10-8264-9531.E-mail: wjl@aphy.iphy.ac.cn
}

\begin{abstract}
A grid-shadow effect is observed in grid-enhanced plasma source ion implantation, in which ions produced within the plasma region are extracted through a grid electrode and then accelerated to the inner surface of a cylindrical bore for implantation. By simulating the ion transportation behaviors from the grid electrode to the inner surface using a Monte Carlo model, we find that the grid-shadow effect results from grid blocking of the ion emission on the grid electrode, and it varies with the experimental parameters such as the gap distance between the grid electrode and the inner surface, the gas pressure, and the applied negative potential.
\end{abstract}

\section{Introduction}

Plasma source ion implantation (PSII) or plasma immersion ion implantation (PIII) [1] is an effective technique to enhance surface properties of materials such as metals, polymers, ceramics and semiconductors. In PSII, a target is immersed in a plasma. When a series of negative high-voltage pulses are applied to the target, ions are extracted directly from the plasma and accelerated to the target for implantation. Since this technique was proposed by Conrad et al. in 1987 [2], it has received great attention in recent years [38]. Grid-enhanced plasma source ion implantation (GEPSII) is a newly proposed method in our laboratory to enhance the inner surface properties of a cylindrical bore $[9,10]$. In our method (see Fig. 1), plasma is produced by $\mathrm{rf}$ discharge established between the axial central cathode and the coaxial grounded grid electrode, the plasma can diffuse through the grid electrode to the inner surface of the tubular sample named the target. When a negative voltage pulse is applied on the target, positive ions within the region between the grid electrode and the inner surface can be accelerated into the target. Experimental and theoretical studies [9-13] have demonstrated the advantages of this new technique over other ion implantation techniques for inner surface modification of a cylindrical bore $[5,7,14]$. However, grid-like patterns are observed on the inner surface processed by GEPSII (see Fig. 1), and the grid-line regions correspond to regions with fewer ions implanted, we call it the grid-shadow effect. Obviously, the existence of grid shadow may reduce the implantation uniformity on the inner surface, and experimental studies [15] have shown that the surface characteristics of the shadow and unshadow areas are different, e.g. the surface hardness of the shadow area is weaker than that of the unshadowed area. In this paper, we will investigate the grid-shadow effect by Monte Carlo simulation.

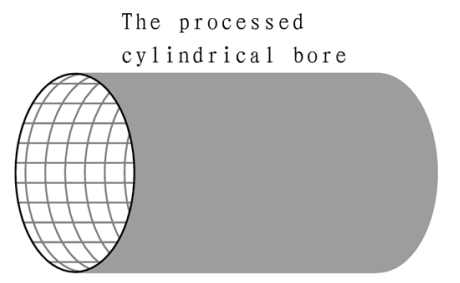

Fig. 1. The grid-shadow effect observed on the inner surface of the processed cylindrical bore by GEPSII (the grid-line regions correspond to the shadow regions)

\section{Model}

In this paper, we select Monte Carlo model to follow the ion trajectories within the region between the grid electrode and the inner surface as illustrated in Fig. 2: the radius of the grid electrode and the inner surface are $r_{\mathrm{a}}$ and $r_{\mathrm{b}}$, respectively, with the gap distance of $d$. The Monte Carlo method used in this paper is similar to that we used in Ref. [16]. Because we are only concerned about the grid-shadow effect here, we simply consider the steady-state ion implantation period when the applied potential and the sheath thickness are fixed, and our simulation region is accordingly the PSII ion sheath region.

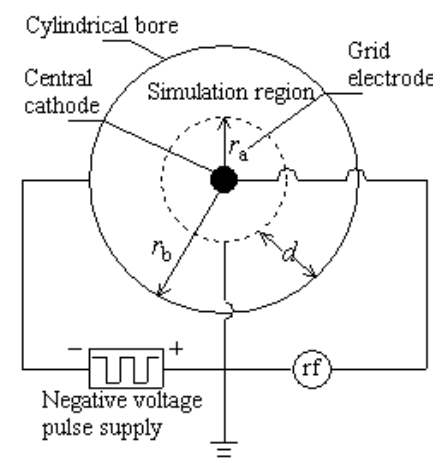

Fig. 2. Cross-section diagram of the GEPSII system considered

Ions start at the grid electrode $\left(r_{0}=r_{\mathrm{a}}\right)$ with initial velocities randomly chosen from a Maxwellian distri- 
bution with the neutral gas temperature $(300 \mathrm{~K})$. To investigate the grid-shadow effect, initial ions at the grid electrode are uniformly placed only on positions corresponding to the grid holes in the experiment, i.e. the ion initial azimuthal angle $\theta_{0}$ is uniformly distributed between $0 \sim 2 \pi$ except some parts corresponding to the grid lines. To reduce the simulation time, only ions with the initial axial position $z_{0}$ of within $0 \sim 10 \mathrm{~mm}$ are considered, also extracting the parts corresponding to the grid lines.

In order to test the validity of our numerical model, we recalculate the ion energy and angle distributions at the inner surface of a large cylindrical bore, which has been calculated by Liu et al. [8] in cylindrical geometry using Monte Carlo model. To investigate the grid-shadow effect, we advance ions in constant time steps $\Delta t$, follow the ion azimuthal angle $\theta$ and axial position $z$ in every time step, but not in constant distance step $\Delta r$ as used in Liu's simulation. Our calculated results under their conditions agree well with what they have given.

\section{Results and Discussions}

To be consistent with our GEPSII experiment, we select $\mathrm{N}^{+}, 2$ as the simulated plasma ions, and all the other parameters such as the gas pressure $(p)$, the negative potential $\left(\phi_{\mathrm{p}}\right)$ and the gap distance between the grid electrode and the target $(d)$ are based on the experimental data. The size of the grid hole is $0.9 \mathrm{~mm} \times$ $\times 0.9 \mathrm{~mm}$ and the diameter of the grid line is $0.1 \mathrm{~mm}$.

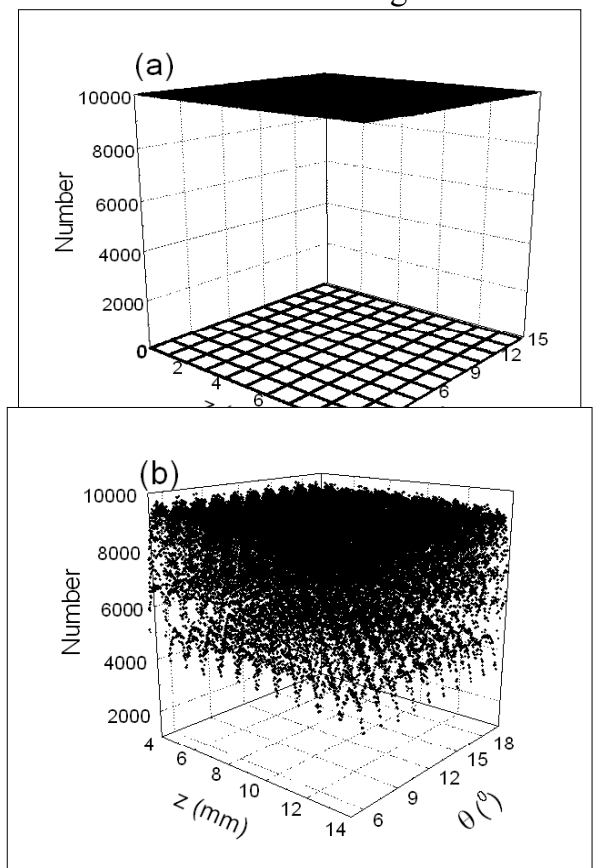

Fig. 3. The initial two-dimensional distribution at the grid electrode (a), and the ion azimuthal angle distribution at the inner surface (b) for $r_{\mathrm{a}}=3.9 \mathrm{~cm}, r_{\mathrm{b}}=5 \mathrm{~cm}, p=0.1 \mathrm{~Pa}$, and

$$
\phi_{\mathrm{p}}=-10 \mathrm{kV}
$$

Figure 3, a shows the two-dimensional initial ion distribution $f\left(\theta_{0}, z_{0}\right)$ at the grid electrode. We divide the grid electrode into uniform small grids $\Delta \theta \times \Delta z$. Initial ions at the grid electrode are uniformly placed only on positions corresponding to the grid holes in the experiment, and there are no ions on positions corresponding to the grid lines. In Fig. 3, b, when all the ions reach the inner surface, the distributions of $f(\theta, z)$ at the grid holes are not uniform any more, and the lower ion distribution regions are the lower ion dose regions and the corresponding shadow regions.

The two-dimensional distribution cannot clearly show the grid-shadow effect. We give the corresponding one-dimensional distribution $f\left(\theta_{0}\right)$ as

$$
f\left(\theta_{0}\right)=\int_{0}^{L} f\left(\theta_{0}, z_{0}\right) \mathrm{d} z,
$$

where $L$ is the length of the grid electrode. $f\left(\theta_{0}\right)$ is normalized such that the integral over the whole azimuthal angle $(0 \sim 2 \pi)$ is 1 . For clarity, only distribution of $\theta_{0}$ in the range of $0^{\circ} \sim 15^{\circ}$ are presented. The uniform ion distribution regions are at angles corresponding to the grid holes, and the regions without ions are at angles corresponding to the grid lines (see Fig. 4, a). When all the initial ions reach the inner surface, the ion azimuthal angle distribution on the inner surface $f\left(\theta_{0}\right)$ can be obtained (see Fig. 4, b). It can be seen that angles with the least ion distributions in Fig. 4, b are rightly the angles with zero ion distributions in Fig. 4, a, and the less ion implanted regions are the corresponding shadow regions, which demonstrates that the show regions source from grid-line blocking of ion emission on the grid electrode. In addition, distributions in the shadow regions are also not uniform, with the minimum values at the center of the shadow regions.

The initial ion axial distribution on the grid $f\left(z_{0}\right)$ and the final ion axial distribution on the target $f(z)$ are not given, because they are similar to the profiles of $f\left(\theta_{0}\right)$ and $f(\theta)$.

During an ion traveling from the grid electrode to the target, it can be accelerated by the radial electric field and scattered due to collisions with the neutral particles. Without collisions, ions will move in line with the electric field along the radial direction, and there will be no ions on the target corresponding to the grid lines, which will be the worst case of the gridshadow effect. When suffering collisions, ions will deviate from their original moving direction, which will be helpful to mitigate the grid-shadow effect on the target. In our experiment, the negative potential is on the order of $-10 \mathrm{kV}$, the gas pressure is about $1.0 \mathrm{~Pa}$, and the radius of the grid electrode is $2.4 \mathrm{~cm}$ or $3.9 \mathrm{~cm}$, with the gap distance of $2.6 \mathrm{~cm} \mathrm{or}$ $1.1 \mathrm{~cm}$. Under such conditions, the effect of electric field acceleration greatly exceeds the effect of collision scattering. The ion movement cannot deviate much from the radial direction, and so the gridshadow effect cannot be avoided. 


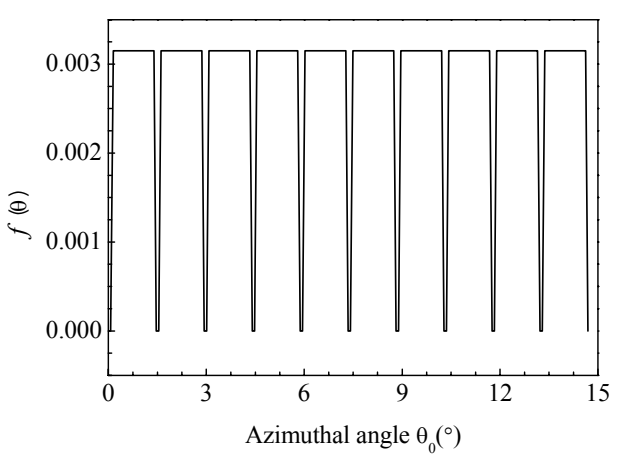

a

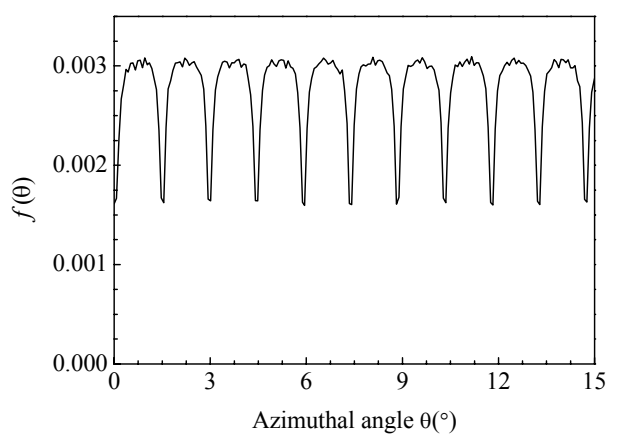

b

Fig. 4. The initial ion azimuthal angle distribution at the grid electrode (a), and the ion azimuthal angle distribution at the inner surface (b) for $r_{\mathrm{a}}=3.9 \mathrm{~cm}, r_{\mathrm{b}}=5 \mathrm{~cm}, p=0.1 \mathrm{~Pa}$, and $\phi_{\mathrm{p}}=-10 \mathrm{kV}$. For clarity, $\theta$ only in the range of $0^{\circ} \sim 15^{\circ}$ are considered

By calculate $f(\theta)$ for different experimental parameters, we find that lower negative potential $\left(\phi_{\mathrm{p}}\right)$, higher gas pressure $(p)$ and longer gap distance $(d)$ can weaken the grid-shadow effect, due to the fact that increasing the negative potential can improve the electric field acceleration, and raising the gas pressure and the ion travel distance can lead to more ion collisions before implantation.

\section{Conclusions}

We have investigated the grid-shadow effect observed in the GEPSII experiment for inner surface modification of a cylindrical bore by Monte Carlo simulation. Typical calculated results such as the ion azimuthal angle distribution on the inner surface of the bore can clearly show the grid-shadow effect. By varying the external parameters in the calculation, it is found that larger gap distance, higher gas pressure, and lower negative potential can reduce the gridshadow effect, which is consistent with the corresponding experimental results. However, it should be pointed out that, such selection of parameters for mitigating the grid-shadow effect are undesirable for improving the ion impact energy [12], and will accordingly reduce the surface hardness of the processed sample [15]. Now we are revising our experimental devices to reduce the grid-shadow effect, e.g. changing the grid electrode into lines parallel to the axial direction and making the cylindrical bore rotating.

\section{Acknowledgments}

This work is supported by the National High Technology Research and Development Program of China (863 Program) under Grant No. 2002A331020, the National Natural Science Foundation of China under Grant Nos. 50071068 and 10275088, and the Science and Technology program of Beijing Municipal Science and Technology Commission under Grant No. H020420160130.

\section{References}

[1] A. Anders, Handbook of Plasma Immersion Ion Implantation and Deposition, New York, Wiely, 2000.

[2] J.R. Conrad, J.L. Radtke, R.A. Dodd, F.J. Worzala, and N.C. Tran, J. Appl. Phys. 62, 4591 (1987).

[3] M.A. Lieberman, J. Appl. Phys. 66, 2926 (1989).

[4] G.A. Emmert, M.A. Henry, J. Appl. Phys. 71, 113 (1992).

[5] T.E. Sheridan, J. Appl. Phys. 74, 4903 (1993).

[6] K.C. Walter, J.T. Scheuer, P.C. Mcintyre, N. Yu., and M. Nastasi, Surf. Coat. Tech. 85, 1 (1996).

[7] X.C. Zeng, T.K. Kwok, A.G. Liu, P.K. Chu, and B.Y. Tang, J. Appl. Phys. 83, 44 (1998).

[8] C.S. Liu, D.Z. Wang, J. Appl. Phys. 91, 32 (2002).

[9] B. Liu, G.L. Zhang, D.J. Cheng, C.Z. Liu, R. He, and S.Z. Yang, J. Vac. Sci. Technol. A 19, 2958 (2001).

[10] B. Liu, C.Z. Liu, D.J. Cheng, G.L. Zhang, R. He, and S.Z. Yang, Nuc. Instrum. Meth. Phys. Res. B 184, 644 (2001).

[11] J.L. Wang, G.L. Zhang, S.H. Fan, W.B. Yang, and S.Z. Yang, Chin. Phys. Lett. 19, 1473 (2002).

[12] J.L. Wang, G.L. Zhang, S.H. Fan, W.B. Yang, and S.Z. Yang, J. Phys. D: Appl. Phys. 361192 (2003).

[13] G.L. Zhang, J.L. Wang, W.B. Yang, S.H. Fan, C.Z. Liu, and S.Z. Yang, Acta Phys. Sin. (in Chinese) 52, 2213 (2003).

[14] M. Sun, S.Z. Yang, and X.C. Chen, J. Vac. Sci. Technol. A 143071 (1996).

[15] G.L. Zhang, J.L. Wang, Y.F. Liu, W.B. Yang, S.H. Fan, C.Z. Liu, and S.Z. Yang, Chin. Phys. Lett. (accepted).

[16] J.L. Wang, G.L. Zhang, Y.N. Wang, Y.F. Liu, and S.Z. Yang, Surf. Coat. Technol. (in press). 